\section{Requirement of protein phosphatase 5 in DNA-damage-induced ATM activation}

\author{
Ambereen Ali, ${ }^{1,4}$ Ji Zhang, ${ }^{1,4}$ Shideng Bao, ${ }^{1}$ \\ Irene Liu, ${ }^{1}$ Diane Otterness, ${ }^{2}$ Nicholas $M$. Dean, ${ }^{3}$ \\ Robert T. Abraham, ${ }^{2}$ and Xiao-Fan Wang ${ }^{1,5}$ \\ ${ }^{1}$ Department of Pharmacology and Cancer Biology, Duke \\ University Medical Center, Durham, North Carolina 27710, \\ USA; ${ }^{2}$ The Burnham Institute, La Jolla, California 92037, \\ USA; ${ }^{3}$ Department of Functional Genomics, GeneTrove \\ (A Division of Isis Pharmaceuticals), Carlsbad, California \\ 92008, USA
}

The checkpoint kinase ATM is centrally involved in the cellular response to DNA double-strand breaks. However, the mechanism of ATM activation during genotoxic stress is only partially understood. Here we report a direct regulatory linkage between the protein serinethreonine phosphatase 5 (PP5) and ATM. PP5 interacts with ATM in a DNA-damage-inducible manner. Reduced expression of PP5 attenuated DNA-damage-induced activation of ATM. Expression of a catalytically inactive PP5 mutant inhibited the phosphorylation of ATM substrates and the autophosphorylation of ATM on Ser 1981, and caused an S-phase checkpoint defect in DNA-damaged cells. Together our findings indicate that PP5 plays an essential role in the activation and checkpoint signaling functions of ATM in cells that have suffered DNA double-strand breaks.

Supplemental material is available at http://www.genesdev.org.

Received December 2, 2003; revised version accepted December 22, 2003.

Eukaryotic cells have evolved cell cycle checkpoints that control cell cycle progression and DNA repair to maintain genomic DNA integrity after cellular exposure to genotoxic stress (Zhou and Elledge 2000; Abraham 2001). ATM is a serine-threonine kinase that phosphorylates several critical checkpoint proteins, including human Rad17 (hRad17), Chk2, p53, BRCA1, and NBS1, in response to ionizing irradiation (IR) or other genotoxic agents that trigger DNA double-strand breaks (DSBs; for review, see Kastan and Lim 2000; Shiloh 2003). Biallelic loss-of-function mutations in the human ATM gene give rise to ataxia telangiectasia (A-T), an autosomal recessive disease characterized by neuronal and immune dysfunction, cancer predisposition, and premature aging

[Keywords: DNA damage; cell cycle checkpoint; ATM; protein phosphatase 5]

${ }^{4}$ These authors contributed equally to this work.

${ }^{5}$ Corresponding author.

E-MAIL wang0011@mc.duke.edu; FAX (919) 681-7152.

Article and publication are at http://www.genesdev.org/cgi/doi/10.1101/ gad.1176004
(Savitsky et al. 1995). A-T cells display characteristic defects in the functions of the $G_{1}, S$, and $G_{2}$ checkpoints following cellular exposure to IR (Zhou and Elledge 2000; Abraham 2001). Irradiated A-T cells also exhibit increased levels of chromosomal breakage, telomere fusions, and significantly heightened sensitivity to killing by IR (Kastan and Lim 2000; Abraham 2001).

The protein kinase activity of immunoprecipitated ATM increases by several fold within $1 \mathrm{~h}$ of cellular exposure to IR or radiomimetic agents (Abraham 2001). However, very little is known about the molecular mechanism by which ATM senses and responds to IRinduced DNA damage. One possibility is that, like many conventional protein kinases, ATM undergoes a stimulus-induced autophosphorylation event that increases its phosphotransferase activity toward heterologous substrates. An autophosphorylation site, at serine 1981 of ATM, has recently been identified, and the functional significance of the modification of this site has been documented (Bakkenist and Kastan 2003). Ser 1981 is rapidly phosphorylated in response to low doses of IR, leading to the dissociation of dimeric/multimeric ATM complexes, and, in turn, the release of activated, monomeric ATM polypeptides (Bakkenist and Kastan 2003). This report also presented intriguing evidence that epigenetic events, for example, chromatin structural perturbations induced by DSBs, serve as the actual trigger for ATM activation. However, it is not clear whether ATM activation requires other critical steps in addition to the autophosphorylation on Ser 1981 that are catalyzed by still unknown protein kinases or phosphatases.

Intracellular signaling cascades are often regulated by the counterbalancing activities of protein kinases and phosphatases. The protein kinases that participate in checkpoint signaling pathways have drawn the most attention, but the possibility that protein phosphatases are also involved in regulating the timing and magnitude of checkpoint activation responses remains unexplored. In this report, we identified protein phosphatase 5 (PP5) as a crucial regulator of ATM kinase activity in response to IR-induced DNA damage. PP5 is a member of the protein serine/threonine phosphatase family, including PP1, PP2A, and PP2B (Chen et al. 1994). It contains a C-terminal catalytic domain and an N-terminal tetratricopeptide repeats (TPR) domain that mediates its interaction with several proteins, including glucocorticoid-receptorheat-shock protein 90 heterocomplexes (Silverstein et al. 1997), CDC16 and CDC27 (Ollendorff and Donoghue 1997), apoptosis signal-regulating kinase 1 (Morita et al. 2001), A subunit of PP2A (Lubert et al. 2001), and G $\alpha_{12}$ and $\mathrm{G} \alpha_{13}$ (Yamaguchi et al. 2002). Unlike its related members, PP5 is less abundant and its basal activity is extraordinarily low under typical protein phosphatase assay conditions (Chinkers 2001). The TPR domain and a region at the $\mathrm{C}$ terminus negatively regulate PP5 (Chen and Cohen 1997; Sinclair et al. 1999), whereas polyunsaturated fatty acids and CoA esters stimulate its activity (Chen and Cohen 1997; Ramsey and Chinkers 2002). However, how PP5 is activated is unclear, and the in vivo targets of activated PP5 remain to be identified.

In this report, we identify PP5 as an ATM-interacting 
protein and demonstrate that PP5 is required for the activation of ATM and subsequent phosphorylation of downstream target proteins. Importantly, the autophosphorylation of ATM on Ser 1981, which has been shown as a direct indicator for the activation of ATM (Bakkenist and Kastan 2003), was significantly reduced in cells that expressed a dominant-negative mutant form of PP5, suggesting a critical role for PP5 in the initial phase of signal relay leading to the activation of the ATM-dependent checkpoint pathway.

\section{Results and Discussion}

\section{DNA damage regulates the interaction} between PP5 and ATM

In a previous study, we demonstrated that the ATM/ ATR-dependent phosphorylation of human $\operatorname{Rad17}$ is a critical step for checkpoint activation in response to DNA damage (Bao et al. 2001). To further investigate the hRad17-mediated checkpoint functions, we searched for interactions between hRad17 and other signaling proteins. In a yeast two-hybrid screen using hRad17 as the bait, we isolated a cDNA clone encoding the full-length PP5, a serine-threonine phosphatase. To confirm that the interaction between hRad17 and PP5 occurred in mammalian cells, we treated HEK 293T cells with or without the radiomimetic agent, neocarzinostatin (NCS), and performed a coimmunoprecipitation assay. As shown in Figure 1A, endogenous hRad17 was found in the $\alpha$-PP5 immunoprecipitates, and interestingly, the association between these two proteins was reduced af-

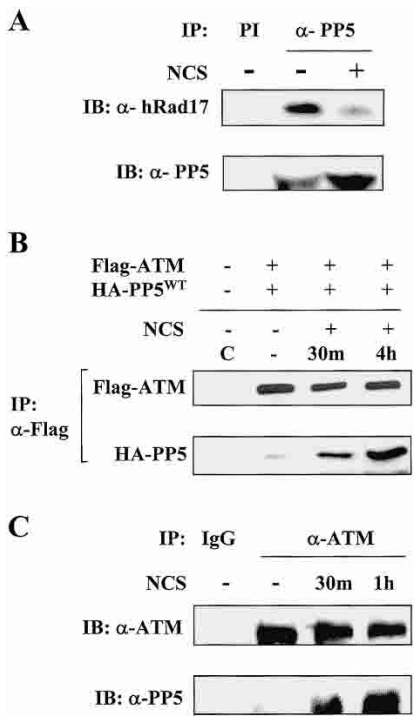

Figure 1. Association of PP5 with ATM and hRad17. (A) Coimmunoprecipitation of endogenous PP5 and hRad17. HEK 293T cells were left untreated or treated with $100 \mathrm{ng} / \mathrm{mL} \mathrm{NCS} \mathrm{for} 30 \mathrm{~min}$ prior to lysis. Preimmune serum (PI) was used in control immunoprecipitation as indicated. $(B)$ DNA-damage-induced association of ectopically expressed PP5 ${ }^{\mathrm{WT}}$ and ATM in HEK 293T cells was determined by $\alpha$-Flag immunoprecipitation followed by $\alpha$-HA immunoblotting (lower panel). (C) Association of endogenous ATM with PP5 was determined by immunoprecipitation assays with $\alpha$-ATM monoclonal antibody with or without treatment of NCS as indicated. Normal mouse $\operatorname{IgG}(\mathrm{IgG})$ was used in the control immunoprecipitation. ter NCS treatment. This result suggests that these two molecules are indeed associated in vivo.

Previously, we demonstrated that hRad17 interacts with ATM in a DNA-damage-inducible manner (Bao et al. 2001). To determine whether PP5 also interacts with ATM, we immunoprecipitated epitope-tagged PP5 and ATM from untreated or NCS-treated HEK 293T cells. As shown in Figure 1B, the ectopically expressed ATM was found to associate with PP5, and this interaction was apparently increased after the cells were exposed to NCS. The same results were obtained with cells exposed to 20 Gy IR (data not shown). To confirm the observed ATM-PP5 interaction under more physiological conditions, we examined the association between endogenous ATM and PP5 in nontransfected cells. As shown in Figure 1C, PP5 was readily detected in a complex with ATM in the $\alpha$-ATM immunoprecipitates. Consistent with the results from Figure 1B, the interaction between PP5 and ATM increased 30 min after NCS treatment. The DNAdamage-induced association between ATM and PP5 suggests that there is a regulatory linkage between ATM and PP5.

\section{Down-regulation of PP5 attenuates DNA-damage-induced ATM activation}

To investigate the functional significance of the DNAdamage-regulated ATM-PP5 interaction, we adopted an antisense oligonucleotide-based approach that was used previously to suppress PP5 expression in mammalian cells (Zuo et al. 1998). Human lung carcinoma-derived A549 cells were transfected with PP5-targeting antisense oligonucleotides or mismatch control oligonucleotides. At $24 \mathrm{~h}$ posttransfection, the cells were exposed to IR, and PP5 expression levels were determined by immunoblotting. As shown in the top panel of Figure 2A, PP5 protein was almost completely eliminated in the antisense oligonucleotide-treated cells (lanes 5 and 6). In control cells, the phosphorylation of hRad17 at Ser 635 was induced at $30 \mathrm{~min}$ after exposure to IR (Fig. 2A, second panel, lanes 1 and 2). In contrast, the DNA-damage-induced phosphorylation of hRad17 was almost completely abrogated in cells that were treated with the PP5 antisense oligonucleotides (Fig. 2A, second panel, lanes 5 and 6). Our initial assumption was that PP5 would act as a negative regulator of the ATM-mediated checkpoint pathway by dephosphorylating ATM substrates. Indeed, in vitro biochemical assays confirmed that recombinant PP5 was capable of dephosphorylating the phosphorylated Ser 635 residue in hRad17 (Supplemental Fig. 2B). Contrary to our expectations, the above results indicated that suppression of PP5 attenuated the DNA-damage-induced hRad17 phosphorylation.

Because ATM is primarily responsible for phosphorylation of hRad17 at Ser 635 in IR-damaged cells (Bao et al. 2001), we determined whether the activity of ATM was altered in PP5 down-regulated cells in response to DNA damage. In the control or mismatch oligonucleotidetransfected cells, IR treatment provoked an increased level of ATM kinase activity (3.6-fold and 3.3-fold increase, respectively) as measured in immune complex kinase assays with GST-p53(1-70) as the substrate (Fig. 2A, fourth panel, lanes 2 and 4). However, transfection of cells with PP5 antisense oligonucleotides effectively reduced protein levels of PP5, and concomitantly sup- 

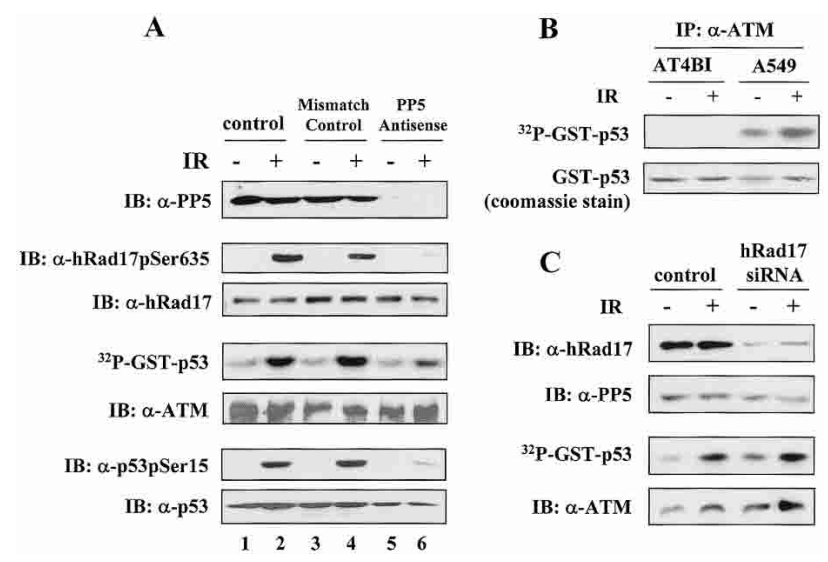

Figure 2. PP5 antisense attenuates DNA-damage-induced ATM kinase activity in A549 cells. (A) After transfection with PP5 antisense or mismatch control oligonucleotides, A549 cells were exposed to IR (20 Gy) and were harvested after $30 \mathrm{~min}$. Cellular extracts were immunoprecipitated with $\alpha$-ATM (Ab3) antibody, and ATM kinase assays were performed with GST-p53(1-70) as the substrate. Amounts of hRad17pSer635, total hRad17, p53pSer15, total p53, and PP5 were determined by immunoblotting with the respective antibodies and were shown in the indicated panels. IR treatment led to an increased level of ATM kinase activity in control, mismatch, or antisense oligonucleotide-transfected cells by 3.6-, 3.3-, and 1.3fold, respectively. $(B)$ The specificity of the ATM antibody used in the immune complex kinase assays were confirmed in A-T cells (AT4BI) and A549 cells. (C) Suppression of hRad17 expression by synthetic siRNA duplexes has no effect on ATM kinase activity. A549 cells were mock transfected or transfected with hRad17 siRNA for $48 \mathrm{~h}$. Cells were then exposed to $20 \mathrm{~Gy}$ of IR followed by 30 min of incubation. The ATM kinase activity and expression levels of hRad17 and PP5 were determined. Consistent results were obtained among three independent experiments.

pressed the activation of ATM kinase after IR exposure (Fig. 2A, lanes 5 and 6). The suppressive effects of the PP5 removal on ATM function were not explained by a reduction in the level of ATM protein (Fig. 2A, fifth panel). The cells with reduced PP5 also displayed severe reductions in IR-dependent phosphorylation of endogenous p53 at Ser 15, a known ATM phosphorylation site (Fig. 2A, sixth panel, lanes 5 and 6). The specificity of the ATM antibodies used in the immune complex kinase assays was confirmed in A-T cells (Fig. 2B). These findings indicate that PP5 is required for ATM kinase activation and subsequent phosphorylation of ATM substrates in IR-damaged cells.

The interaction between PP5 and hRad17 prompted us to investigate whether hRad17 is also required for ATM kinase activation. To address this question, we used small-interfering RNA (siRNA) to specifically inhibit the expression of hRad17 in A549 cells. As shown in Figure 2C, introduction of hRad17-targeted siRNA duplexes led to a significant decrease in the hRad17 protein level (top panel). The expression of PP5 was shown as the loading control (Fig. 2C, second panel). The kinase activity of ATM was then determined in the same cell lysates. As shown in the third panel of Figure 2C, ATM was activated by IR in hRad17 knockdown cells, and the level of activation was comparable to that obtained from control cells (these results are further supported by data presented in Supplemental Fig. 1, demonstrating that the phosphorylation of Ser 1981 on ATM, an indicator for ATM activation, is not affected by down-regulation of hRad17). These results suggest that hRad17, unlike PP5, is not required for the activation of ATM kinase activity, and therefore, it is unlikely that the functional regulation of PP5 on ATM activation is mediated by hRad17. At present, the functional significance of DNA-damagerepressed association between hRad17 and PP5 remains unclear.

\section{Expression of a catalytically inactive PP5 mutant inhibits ATM activation}

As an alternative approach to probe the functional relationship between PP5 and ATM activation, we determined whether expression of a catalytically inactive form of PP5 interferes with the activation of ATM. Previously, the $\mathrm{C}$-terminal region of PP5 was shown to regulate its nuclear localization, and a PP5 mutant lacking residues 315-419 in the phosphatase catalytic domain but with intact nuclear localization sequence was found to localize in both the nucleus and the cytoplasm as was seen with the wild-type protein (Borthwick et al. 2001). In light of these results and the fact that the nucleus is the subcellular compartment where the DNA damage response initiates, we generated the identical catalytically inactive PP5 mutant (PP5 ${ }^{\mathrm{MT}}$; Fig. 3A). After obtaining purified GST fusion proteins of both wild-type and mutant PP5, we found that the recombinant PP5 ${ }^{\mathrm{MT}}$ protein retained the ability to interact with hRad17, but it failed to dephosphorylate the Ser 635 site in hRad17 in an in vitro phosphatase assay (Supplemental Fig. 2A,B). We also determined the binding affinity between ATM and PP5 ${ }^{\mathrm{WT}}$ or PP5 ${ }^{\mathrm{MT}}$ by cotransfecting HEK $293 \mathrm{~T}$ cells with Flag-ATM and HA-tagged PP5 ${ }^{\mathrm{WT}}$ or $\mathrm{PP} 5^{\mathrm{MT}}$. As

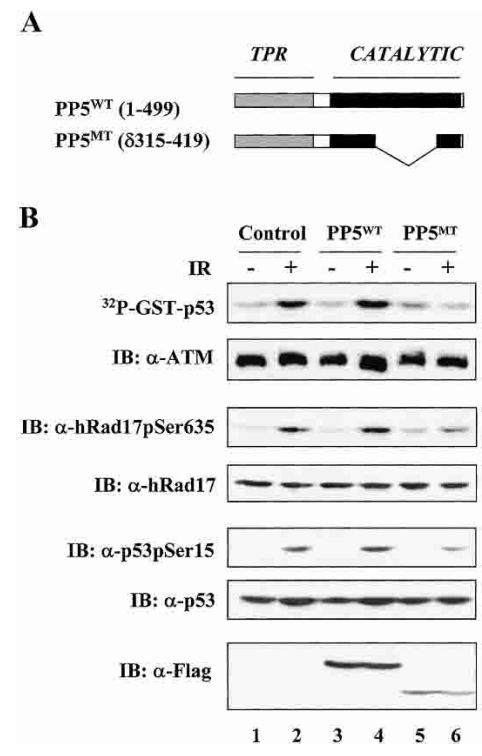

Figure 3. Expression of a catalytically inactive form of PP5 (PP5 $\left.{ }^{\mathrm{MT}}\right)$ inhibits ATM kinase activity. (A) A mutant form of PP5 was generated by deleting residues $315-419$ of the catalytic domain. $(B)$ Overexpression of PP5 ${ }^{\mathrm{MT}}$ attenuates ATM kinase activity. After infection by recombinant adenoviruses, A549 cells were exposed to IR $(20 \mathrm{~Gy})$ and then incubated for $30 \mathrm{~min}$. ATM immune complex kinase assays were performed with $1 \mu \mathrm{g}$ of GST-p53(1-70) as the substrate. Cell lysates were immunoblotted as indicated. IR treatment led to an increased level of ATM kinase activity in control cells or PP5 ${ }^{\mathrm{WT}} / \mathrm{PP}^{\mathrm{MT}}$-expressing cells by $2.9-, 2.1-$, and 0.8 -fold, respectively. Consistent results were obtained among three independent experiments. 
shown in Supplemental Figure 2C, both PP5 WT and $\mathrm{PP} 5^{\mathrm{MT}}$ coimmunoprecipitated with FlagATM; however, a relatively higher amount of $\mathrm{PP}^{\mathrm{MT}}$ bound to ATM was recovered. Therefore, this catalytically inactive PP5 mutant was likely to have a dominant-interfering effect in vivo. To examine the effect of PP5 ${ }^{\mathrm{MT}}$ on ATM activation, A549 cells were infected with control or recombinant adenovirus encoding either PP5 ${ }^{\mathrm{WT}}$ or $\mathrm{PP}^{\mathrm{MT}}$. At $24 \mathrm{~h}$ postinfection, the cells were harvested and ATM kinase activity was assessed with GST-p53(1-70) as the substrate (Fig. 3B, top panel). A549 cells that were infected with the control adenovirus showed a pronounced activation of ATM following exposure to IR (Fig. 3B, lane 2). Interestingly, cells expressing PP5 ${ }^{\mathrm{WT}}$ displayed a similar phenotype as those infected with the control virus (Fig. 3B, lane 4). This result was in agreement with previous observations that the basal phosphatase activity of PP5 is extremely low (Chinkers 2001) and PP5 may be regulated by an unknown mechanism. In contrast, A549 cells infected with the mutant PP5-encoding adenovirus failed to activate ATM after IR exposure (Fig. $3 \mathrm{~B}$, lane 6 , top panel). Consistent with this observation, IR-induced phosphorylation of endogenous hRad17 and p53, two key ATM substrates, was also reduced in the PP5 ${ }^{\mathrm{MT}}$-expressing cells (Fig. 3B, lane 6, third and fifth panels). The amounts of overexpressed PP5 ${ }^{\mathrm{WT}}$ and PP5 $5^{\mathrm{MT}}$ were determined and shown in the bottom panel. These results further support a role for PP5 in the ATM activation process.

PP5 activity is required for IR-induced S-phase checkpoint activation

To determine whether the phenomenon observed above can be generalized to other cell types, we examined the impact of loss of PP5 functions on ATM-dependent checkpoint signaling in human diploid BJ fibroblasts. In the initial studies, we confirmed that loss of PP5 resulted in the abrogation of DNA-damage-induced hRad17 and p53 phosphorylation, as observed in A549 cells. In this case, PP5 expression was down-regulated in BJ fibroblasts with siRNA specific for PP5. As shown in Figure 4A (top panel), transfection of the BJ cells with synthetic siRNA duplexes reduced PP5 protein levels by $90 \%$. Under the condition of reduced PP5 expression, the IR-induced phosphorylation of endogenous p53 and hRad17 was severely impaired (Fig. 4A, second and fourth panels, respectively). To examine the effect of PP5 inhibition on IR-induced S-phase checkpoint activation, BJ fibroblasts were infected with control or recombinant adenovirus encoding PP5 ${ }^{\mathrm{WT}}$ or PP5 ${ }^{\mathrm{MT}}$. At $24 \mathrm{~h}$ postinfection, the cells were either left untreated or exposed to IR. The infected cells expressed comparable levels of wild-type or mutant proteins (Fig. 4C). The DNA synthesis rates were determined by a ${ }^{3} \mathrm{H}$-thymidine incorporation assay. Uninfected BJ cells or cells that were infected with either control or PP5 ${ }^{\mathrm{WT}}$-expressing adenoviruses showed a pronounced inhibition of DNA synthesis following exposure to IR. In contrast, the inhibition of DNA synthesis in cells infected with the PP5 ${ }^{\mathrm{MT}}$-encoding adenovirus was significantly compromised, indicating that inhibition of PP5 function resulted in a radioresistant DNA
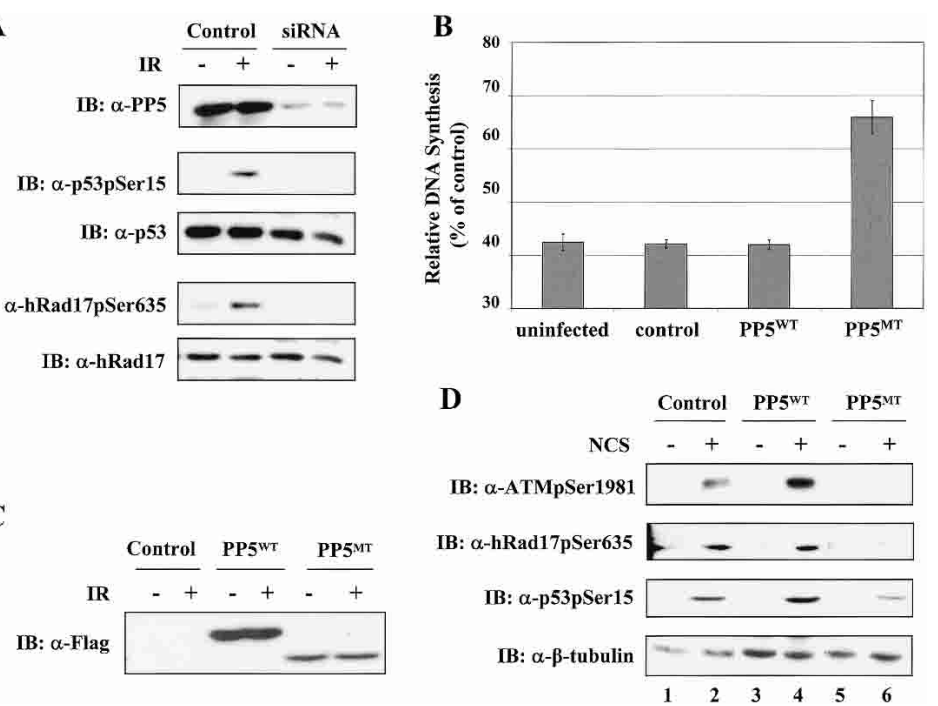

igure 4. PP5 activity is required for IR-induced S-phase checkpoint activation human BJ fibroblasts. (A) Down-regulation of PP5 by synthetic siRNA dulexes abrogates IR-induced phosphorylation of p53 and hRad17. After transfection, BJ cells were exposed to IR (20 Gy) and then harvested after $30 \mathrm{~min}$. Cellar extracts were analyzed by immunoblotting with the indicated antibodies. B) PP5 ${ }^{\mathrm{MT}}$ induces radioresistant DNA synthesis in BJ cells. After infection with the indicated adenoviruses, BJ cells were exposed to $20 \mathrm{~Gy}$ of IR, and DNA ynthesis rates were determined as described in Materials and Methods. (C) A fraction of the cells from $B$ was analyzed by immunoblotting with $\alpha$-Flag antibody. (D) PP5 ${ }^{\mathrm{MT}}$ inhibits the autophosphorylation of ATM on Ser 1981. Adenoirus-infected BJ cells were either left untreated or treated with $100 \mathrm{ng} / \mathrm{mL} \mathrm{NCS}$ or $30 \mathrm{~min}$. Cells were then harvested and analyzed by immunoblotting with $\alpha$-ATMpSer1981, $\alpha$-hRad17pSer635, and $\alpha$-p53pSer15. $\beta$-Tubulin was used as a oading control. Consistent results were obtained among three independent experiments.

synthesis (RDS) phenotype (Fig. 4B). Because the RDS defect is a hallmark feature of ATM-deficient cells, these results are consistent with the idea that ATM and PP5 reside within a common checkpoint signaling pathway.

\section{Interference of PP5 function led to reduction in ATM autophosphorylation on Ser 1981}

A recent study demonstrated that the Ser 1981 residue on ATM was rapidly phosphorylated after irradiation, and this phosphorylation was intimately associated with the activation of ATM-dependent checkpoint in IR-damaged cells (Bakkenist and Kastan 2003). To determine whether PP5 regulates the initial phase of the ATM activation process, we examined the effect of the dominant-interfering activity of PP5 mutant on ATM autophosphorylation of Ser 1981. As shown in the top panel of Figure 4D, expression of the PP5 mutant profoundly inhibited the NCS-induced autophosphorylation of ATM on Ser 1981 in BJ fibroblasts (lanes 5 and 6). Similar results were observed in IR-damaged cells (data not shown). As a consequence of the ATM activation defect, the phosphorylation of endogenous hRad17 and p53 was also significantly reduced in the PP5 ${ }^{\mathrm{MT}}$-expressing cells (Fig. 4D, second and third panels). Together, these data indicated that PP5 activity is directly involved in the early phase of IR-induced ATM activation.

In summary, we have identified a direct regulatory linkage between the serine-threonine phosphatase PP5 and the checkpoint kinase ATM. Down-regulation of 
PP5 by antisense or siRNA attenuated the kinase activity of ATM after IR exposure, and reduced phosphorylation of the known ATM substrates, hRad17 and p53. The physiological significance of the interaction between PP5 and ATM was further supported by the observation that ectopic expression of a mutant PP5 that interferes with the function of endogenous PP5 caused RDS, one of the characteristic phenotypes of ATM-deficient cells. An intriguing insight into the role of PP5 in the ATM activation process was provided by the finding that phosphorylation of the positive regulatory Ser 1981 site in ATM was significantly inhibited by the presence of the same mutant PP5. Taken together, these results strongly suggest that PP5 is an important regulator of ATM activation during the initial phase of checkpoint activation in response to DNA damage.

The protein kinase activity of ATM increases by several folds at early times after IR exposure. However, the mechanism by which ATM becomes activated has remained poorly understood. Posttranslational modifications, such as phosphorylation, have been suggested to play important roles in this process. Recent studies demonstrate that "inactive" ATM exists as a dimer or higher-order oligomer, and that IR exposure induces the trans-phosphorylation of Ser 1981 in ATM, an event triggering the disassembly of ATM homomeric complexes into monomeric ATM. The increase in protein kinase activity observed in $\alpha$-ATM immunoprecipitates from IR-treated cells reflects the increased abundance of ATM monomers (Bakkenist and Kastan 2003). Notably, the mutation of Ser 1981 has no direct effect on ATM catalytic activity in vitro, suggesting the existence of other regulatory mechanisms for the activation of ATM kinase. Taken together with the results of the present study, those findings suggest that the catalytic activity of ATM is normally repressed by serine/threonine phosphorylation at a site(s) other than Ser 1981, and that PP5 removes these inhibitory phosphates in response to IR-induced DNA damage. This mode of activation of a protein kinase by a phosphatase is not without precedent. DNA-PK, an ATMrelated kinase, as well as GSK3 and Cdk2, are activated by protein phosphatases (Norbury et al. 1991; Cohen and Frame 2001; Douglas et al. 2001). Interestingly, dephosphorylation of ATM after irradiation was also observed by tryptic phosphopeptide mapping in a recent study, although the identity of the dephosphorylated site(s) is not yet known (Kozlov et al. 2003). At present, however, we cannot rule out the possibility that PP5 acts on a regulatory protein associated with ATM, rather than ATM itself. In view of the recent report that the Mre11Rad50-Nbs1 (MRN) is involved in the initial phase of ATM activation (Uziel et al. 2003), it will be interesting to determine whether there is a regulatory linkage between PP5 and the MRN complex.

It is intriguing to consider the role of $\operatorname{hRad} 17$ in the formation of the PP5-ATM complex. We originally hypothesized that hRad17 might facilitate the interaction between PP5 and ATM at sites of DNA damage. However, as our results suggest, suppression of hRad17 has a minimal direct effect on ATM kinase activity or Ser 1981 phosphorylation of ATM, implying that hRad17 is not mediating the association of PP5 to ATM and the consequent activation of ATM. It is of interest to note that such a role of hRad17 to ATM is parallel to the previous finding that the formation of UV-induced ATR foci is largely independent of hRad17 (Zou et al. 2002). In this regard, it is puzzling that hRad17 quickly dissociated from PP5 after NCS treatment when PP5 was recruited to ATM. At the present stage, it is unclear whether the same or different pools of PP5 molecules are interacting with hRad17 and ATM in undamaged versus damaged cells. It is possible that the hRad17-bound fraction of PP5 has a distinct role in a later stage of the DNA damage response, such as dephosphorylating hRad17 as a feedback regulatory mechanism. Regardless, further studies of the interplay between PP5 and its two interacting proteins, ATM and hRad17, are clearly warranted. Furthermore, the present findings suggest that specific inhibitors of PP5 could represent a novel class of radioand chemo-sensitizing agents for cancer therapy.

\section{Materials and methods}

Cell culture and antibodies

The A549, HEK 293T cell lines were cultured in DMEM with 10\% FBS. The BJ human fibroblasts were maintained in DMEM containing $20 \%$ FBS. The A-T cell line, AT4BI, was grown in DMEM/F12 supplemented with $20 \%$ FCS. Where indicated, cells were irradiated with a ${ }^{137} \mathrm{Cs}-\gamma$-ray source. Phosphospecific antibodies directed against hRad17 ( $\alpha$ hRad17pSer635) were generated as described previously (Bao et al. 2001). The hRad17 antibody was from Santa Cruz (H-300). The $\alpha$-ATM antibodies were from Oncogene Research Products (Ab-3) and GeneTex Inc. (ATM-2C1). Affinity-purified ATMpSer1981 antibody and phosphop53(Ser15) antibody were from Rockland Immunochemicals and Cell Signaling, respectively. The $\alpha$-Flag-M2 monoclonal antibody was from Sigma. PP5-specific antiserum was generated by immunizing rabbits with a glutathione-S-transferase (GST)-PP5 fusion protein and affinity purified.

Plasmid and adenoviral constructs

The expression vector Flag-hRad17 was constructed as described previously (Bao et al. 2001). The expression plasmid HA-PP5 was constructed by cloning the PCR-amplified HA-tagged PP5 coding sequence into the EcoRI-BamHI sites of the pcDNA3 vector. The expression plasmid FlagPP5 ${ }^{\mathrm{WT}}$ was constructed by cloning the PCR-amplified Flag-tagged PP5 ${ }^{\mathrm{WT}}$ coding sequence into the EcoRI-BamHI sites of the pcDNA3 vector. The expression vector Flag-PP5 ${ }^{\mathrm{MT}}(\Delta 315-419)$ was generated with the QuickChange Mutagenesis kit (Stratagene) according to the manufacturer's protocol. Control adenovirus or recombinant adenovirus encoding Flag$\mathrm{PP}^{\mathrm{WT}}$ or Flag-PP5 ${ }^{\mathrm{MT}}$ was generated and produced as described (He et al. 1998). The Flag-ATM construct has been described previously (Cortez et al. 1999).

Generation of recombinant GST fusion proteins

Glutathione-S-transferase (GST)-PP5 ${ }^{\mathrm{WT}}$ was generated by subcloning PP5 into the EcoRI-XhoI sites of the pGEX-KG GST plasmid. The GSTPP5 ${ }^{\mathrm{MT}}$ was generated by subcloning the PCR-amplified PP5 coding sequence from pCDNA3-FLAG-PP5 ${ }^{\mathrm{MT}}(\Delta 315-419)$ into the EcoRI-Xhol sites of the pGEX-KG GST plasmid. The GST-PP5 proteins were expressed in Escherichia coli, purified, and eluted with glutathione. GSTp53(1-70) was generated as described (Tibbetts et al. 1999).

Antisense oligonucleotide and siRNA transfection

Antisense and mismatch control oligonucleotides targeting PP5 (ISIS 15534 and ISIS 15521) were obtained from Isis Pharmaceuticals and were transfected as described recently (Zuo et al. 1998). Synthetic siRNA duplexes targeting PP5 (AACAUAUUCGAGCUCAACGGU) or hRad17 (AAAGAUGAUUUCAAGGGGAUG) were purchased from Dharmacon Research Inc. A549 cells or BJ cells were transfected with $20 \mu \mathrm{M}$ siRNA duplexes and Oligofectamine (Life Technologies), and were analyzed $48 \mathrm{~h}$ after transfection.

Protein analysis, kinase and phosphatase assays

To examine the association between PP5 and ATM or hRad17, HEK 293T cells were lysed in $50 \mathrm{mM}$ Tris- $\mathrm{HCl}(\mathrm{pH} 7.4), 150 \mathrm{mM} \mathrm{NaCl}, 0.5 \%$ NP-40, $20 \mathrm{mM} \beta$-glycerophosphate, protease inhibitors $(20 \mu \mathrm{g} / \mathrm{mL}$ leupeptin, 10 $\mu \mathrm{g} / \mathrm{mL}$ pepstatin $\mathrm{A}$, and $10 \mu \mathrm{g} / \mathrm{mL}$ aprotonin), $50 \mathrm{nM}$ microcystin-LR, and 
$1 \mathrm{mM}$ DTT. To detect the endogenous PP5-ATM interaction in HEK $293 \mathrm{~T}$ cells, isolated nuclei were collected and resuspended in $50 \mathrm{mM}$ Tris- $\mathrm{HCl}(\mathrm{pH} 7.4), 150 \mathrm{mM} \mathrm{NaCl}, 2 \mathrm{mM} \mathrm{MnSO} 4,1 \mathrm{mM} \mathrm{MgCl}_{2}, 0.5 \%$ NP-40, $20 \mathrm{mM} \beta$-glycerophosphate, protease inhibitors, $50 \mathrm{nM}$ microcystin-LR, and $1 \mathrm{mM} \mathrm{DTT}$. After centrifugation, the nuclear protein-containing supernatant was subjected to immunoprecipitation assays. ATM immune complex kinase assays were performed as described (Abraham 2001), using $1 \mu \mathrm{g}$ of GST-p53(1-70) as the substrate. (Microcystin-LR, a potent serine-threonine phosphatase inhibitor, was included in the above cell lysis buffers as indicated without significant effect on the results of experiments.) PP5 in vitro protein phosphatase assays were performed as the following: HEK 293T cells were transfected with FlaghRad17. After treatment with NCS $(100 \mathrm{ng} / \mathrm{mL})$ for $30 \mathrm{~min}$, cell lysates were immunoprecipitated with $\alpha$-Flag-M2 monoclonal antibody. The immunoprecipitates were then combined with recombinant GST, GSTPP5 ${ }^{\mathrm{WT}}$, or GST-PP5 ${ }^{\mathrm{MT}}$ for $60 \mathrm{~min}$ at $30^{\circ} \mathrm{C}$. Proteins were analyzed by SDS-PAGE and immunoblotting with $\alpha$-hRad17pSer635.

\section{Radioresistant DNA synthesis assay}

Human BJ fibroblasts were incubated with ${ }^{14} \mathrm{C}$-thymidine $(20 \mathrm{nCi} / \mathrm{mL}$, NEN) for $24 \mathrm{~h}$, followed by infection with the indicated recombinant adenoviruses. Then, $24 \mathrm{~h}$ later, the cells were exposed to IR (20 Gy) and incubated with ${ }^{3} \mathrm{H}$-thymidine $(2.5 \mu \mathrm{Ci} / \mathrm{mL}, \mathrm{NEN})$ for $4 \mathrm{~h}$. The cells were then harvested as described (Cliby et al. 1998). The radioactivity was determined by liquid scintillation counting, and the relative DNA synthesis rate was calculated with the equation $\left(\left[{ }^{3} \mathrm{H}\right] /\left[{ }^{14} \mathrm{C}\right]\right)_{20 \text { Gy }} \div([3 \mathrm{H}] /$ $\left.\left[{ }^{14} \mathrm{C}\right]\right)_{0 \mathrm{~Gy}}$.

All samples were tested in triplicate, and consistent results were obtained among three independent experiments.

\section{Acknowledgments}

We thank B. Vogelstein (Johns Hopkins University, Baltimore, MD) for the pAdTrack-CMV and pAdEasy adenoviral vectors, C. Counter (Duke University, Durham, NC) for the BJ fibroblasts, S. Rossie (Purdue University, West Lafayette, IN) and M. Chinkers (University of South Alabama, Mobile, AL) for PP5 antibodies, Y. Yu for technical help, and members of the Wang and Abraham lab for helpful scientific discussions. Grants from the U.S. Army Medical Research Materiel Command (DAMD17-00-1-0228 awarded to A.A; DAMD 17-02-1-0379 awarded to J.Z.; DAMD17-02-1-0730 awarded to R.T.A.), the National Institutes of Health (CA83770, CA93676, and CA97950), and the A-T Children's Project supported this work.

The publication costs of this article were defrayed in part by payment of page charges. This article must therefore be hereby marked "advertisement" in accordance with 18 USC section 1734 solely to indicate this fact.

\section{References}

Abraham, R.T. 2001. Cell cycle checkpoint signaling through the ATM and ATR kinases. Genes \& Dev. 15: 2177-2196.

Bakkenist, C.J. and Kastan, M.B. 2003. DNA damage activates ATM through intermolecular autophosphorylation and dimer dissociation. Nature 421: 499-506.

Bao, S., Tibbetts, R.S., Brumbaugh, K.M., Fang, Y., Richardson, D.A., Ali, A., Chen, S.M., Abraham, R.T., and Wang, X.F. 2001. ATR/ATMmediated phosphorylation of human Rad17 is required for genotoxic stress responses. Nature 411: 969-974.

Borthwick, E.B., Zeke, T., Prescott, A.R., and Cohen, P.T. 2001. Nuclear localization of protein phosphatase 5 is dependent on the carboxyterminal region. FEBS Lett. 491: 279-284.

Chen, M.X. and Cohen, P.T. 1997. Activation of protein phosphatase 5 by limited proteolysis or the binding of polyunsaturated fatty acids to the TPR domain. FEBS Lett. 400: 136-140.

Chen, M.X., McPartlin, A.E., Brown, L., Chen, Y.H., Barker, H.M., and Cohen, P.T. 1994. A novel human protein serine/threonine phosphatase, which possesses four tetratricopeptide repeat motifs and localizes to the nucleus. EMBO J. 13: 4278-4290.

Chinkers, M. 2001. Protein phosphatase 5 in signal transduction. Trends Endocrinol. Metab. 12: 28-32.

Cliby, W.A., Roberts, C.J., Cimprich, K.A., Stringer, C.M., Lamb, J.R.,
Schreiber, S.L., and Friend, S.H. 1998. Overexpression of a kinaseinactive ATR protein causes sensitivity to DNA-damaging agents and defects in cell cycle checkpoints. EMBO J. 17: 159-169.

Cohen, P. and Frame, S. 2001. The renaissance of GSK3. Nat. Rev. Mol Cell. Biol. 2: 769-776.

Cortez, D., Wang, Y., Qin, J., and Elledge, S.J. 1999. Requirement of ATM-dependent phosphorylation of brcal in the DNA damage response to double-strand breaks. Science 286: 1162-1166.

Douglas, P., Moorhead, G.B., Ye, R., and Lees-Miller, S.P. 2001. Protein phosphatases regulate DNA-dependent protein kinase activity. I. Biol. Chem. 276: 18992-18998.

He, T.C., Zhou, S., da Costa, L.T., Yu, J., Kinzler, K.W., and Vogelstein, B. 1998. A simplified system for generating recombinant adenoviruses. Proc. Natl. Acad. Sci. 95: 2509-2514.

Kastan, M.B. and Lim, D.S. 2000. The many substrates and functions of ATM. Nat. Rev. Mol. Cell. Biol. 1: 179-186.

Kozlov, S., Gueven, N., Keating, K., Ramsay, J., and Lavin, M.F. 2003. ATP activates ataxia-telangiectasia mutated (ATM) in vitro. Importance of autophosphorylation. J. Biol. Chem. 278: 9309-9317.

Lubert, E.J., Hong, Y., and Sarge, K.D. 2001. Interaction between protein phosphatase 5 and the A subunit of protein phosphatase 2A: Evidence for a heterotrimeric form of protein phosphatase 5. J. Biol. Chem. 276: 38582-38587.

Morita, K., Saitoh, M., Tobiume, K., Matsuura, H., Enomoto, S., Nishitoh, H., and Ichijo, H. 2001. Negative feedback regulation of ASK1 by protein phosphatase 5 (PP5) in response to oxidative stress. EMBO I. 20: 6028-6036.

Norbury, C., Blow, J., and Nurse, P. 1991. Regulatory phosphorylation of the p34cdc2 protein kinase in vertebrates. EMBO J. 10: 3321-3329.

Ollendorff, V. and Donoghue, D.J. 1997. The serine/threonine phosphatase PP5 interacts with CDC16 and CDC27, two tetratricopeptide repeat-containing subunits of the anaphase-promoting complex. $J$. Biol. Chem. 272: 32011-32018.

Ramsey, A.J. and Chinkers, M. 2002. Identification of potential physiological activators of protein phosphatase 5. Biochemistry 41: 56255632.

Savitsky, K., Bar-Shira, A., Gilad, S., Rotman, G., Ziv, Y., Vanagaite, L., Tagle, D.A., Smith, S., Uziel, T., Sfez, S., et al. 1995. A single ataxia telangiectasia gene with a product similar to PI-3 kinase. Science 268: $1749-1753$.

Shiloh, Y. 2003. ATM and related protein kinases: Safeguarding genome integrity. Nat. Rev. Cancer 3: 155-168.

Silverstein, A.M., Galigniana, M.D., Chen, M.S., Owens-Grillo, J.K., Chinkers, M., and Pratt, W.B. 1997. Protein phosphatase 5 is a major component of glucocorticoid receptor $\cdot$ hsp 90 complexes with properties of an FK506-binding immunophilin. J. Biol. Chem. 272: 1622416230 .

Sinclair, C., Borchers, C., Parker, C., Tomer, K., Charbonneau, H., and Rossie, S. 1999. The tetratricopeptide repeat domain and a C-terminal region control the activity of Ser/Thr protein phosphatase 5. J. Biol. Chem. 274: 23666-23672.

Tibbetts, R.S., Brumbaugh, K.M., Williams, J.M., Sarkaria, J.N., Cliby, W.A., Shieh, S.Y., Taya, Y., Prives, C., and Abraham, R.T. 1999. A role for ATR in the DNA damage-induced phosphorylation of p53. Genes \& Dev. 13: 152-157.

Uziel, T., Lerenthal, Y., Moyal, L., Andegeko, Y., Mittelman, L., and Shiloh, Y. 2003. Requirement of the MRN complex for ATM activation by DNA damage. EMBO J. 22: 5612-5621.

Yamaguchi, Y., Katoh, H., Mori, K., and Negishi, M. 2002. G $\alpha_{12}$ and $\mathrm{G} \alpha_{13}$ interact with Ser/Thr protein phosphatase type 5 and stimulate its phosphatase activity. Curr. Biol. 12: 1353-1358.

Zhou, B.B. and Elledge, S.J. 2000. The DNA damage response: Putting checkpoints in perspective. Nature 408: 433-439.

Zou, L., Cortez, D., and Elledge, S.J. 2002. Regulation of ATR substrate selection by Rad17-dependent loading of Rad9 complexes onto chromatin. Genes \& Dev. 16: 198-208.

Zuo, Z., Dean, N.M., and Honkanen, R.E. 1998. Serine/threonine protein phosphatase type 5 acts upstream of p53 to regulate the induction of p21 $1^{\mathrm{WAF} 1 / \mathrm{Cip} 1}$ and mediate growth arrest. J. Biol. Chem. 273: 1225012258. 


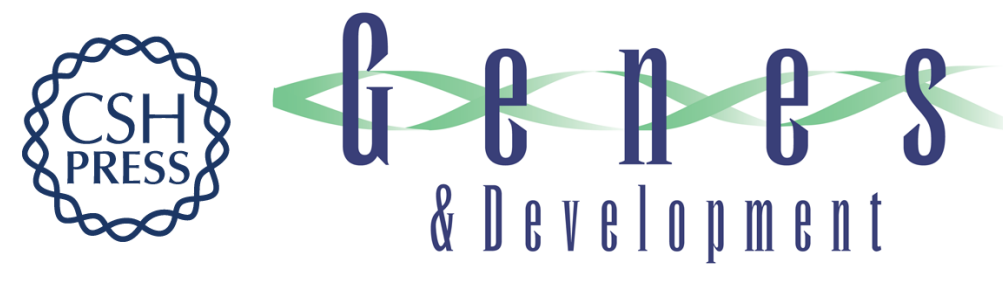

\section{Requirement of protein phosphatase 5 in DNA-damage-induced ATM activation}

Ambereen Ali, Ji Zhang, Shideng Bao, et al.

Genes Dev. 2004, 18:

Access the most recent version at doi:10.1101/gad.1176004

\section{Supplemental http://genesdev.cshlp.org/content/suppl/2004/01/26/18.3.249.DC1 Material}

References This article cites 29 articles, 16 of which can be accessed free at: http://genesdev.cshlp.org/content/18/3/249.full.html\#ref-list-1

\section{License}

Email Alerting

Service

Receive free email alerts when new articles cite this article - sign up in the box at the top right corner of the article or click here.

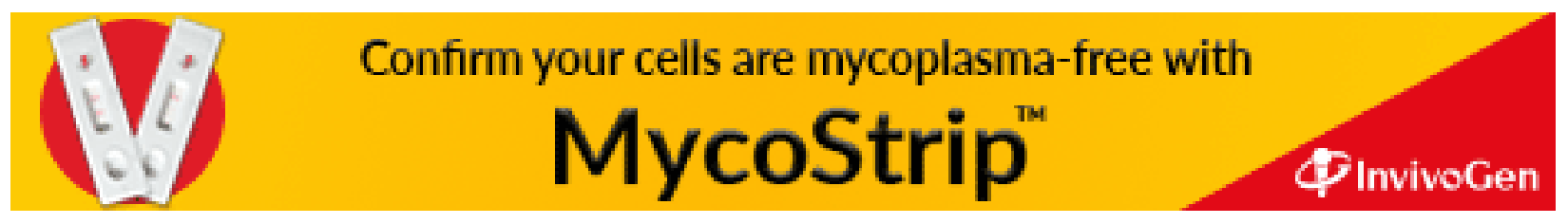

